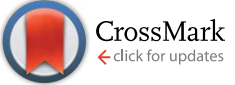

Cite this: RSC Adv., 2017, 7, 8598

Received 8th October 2016 Accepted 11th January 2017

DOI: 10.1039/c6ra24890j

www.rsc.org/advances

\section{Manipulating energy storage characteristics of ultrathin boron carbide monolayer under varied scandium doping $\dagger$}

\begin{abstract}
S. R. Naqvi, ${ }^{a}$ T. Hussain, ${ }^{\star c}$ P. Panigrahi, ${ }^{d}$ W. Luo ${ }^{a}$ and R. Ahuja ${ }^{a b}$
We report, for the first time we believe, a detailed investigation on hydrogen storage efficiency of scandium (Sc) decorated boron carbide $\left(\mathrm{BC}_{3}\right)$ sheets using spin-polarized density functional theory (DFT). We analyzed the energetics of Sc adsorption and explored the most favorable adsorption sites of $\mathrm{Sc}$ on $\mathrm{BC}_{3}$ sheets with $3.12 \%, 6.25 \%$, and $12.5 \%$ coverage effects. Our investigations revealed that Sc strongly binds on pristine $\mathrm{BC}_{3}$ sheet, with a minimum binding energy of $\sim 5 \mathrm{eV}$, which is robust enough to hinder $\mathrm{Sc}-\mathrm{Sc}$ metal clustering. Sc, the lightest transition metal, adsorbs a large number of $\mathrm{H}_{2}$ molecules per atom, resulting in a reasonable storage capacity. With $12.5 \% \mathrm{Sc}$-coverage, functionalized $\mathrm{BC}_{3}$ sheets could attain a $\mathrm{H}_{2}$ storage capacity of $5.5 \mathrm{wt} \%$ with binding energies suitable for a practical $\mathrm{H}_{2}$ storage medium.
\end{abstract}

\section{Introduction}

Hydrogen $\left(\mathrm{H}_{2}\right)$ has emerged as an alternative energy carrier with a strong potential to serve a vibrant economy in the future particularly owing to its application in the transportation sector. ${ }^{1-3}$ However, $\mathrm{H}_{2}$ economy presents challenges when it comes to the fabrication of a solid-state material for $\mathrm{H}_{2}$ storage with high gravimetric/volumetric density that performs efficiently under ambient conditions. ${ }^{4,5}$ Previously, several new materials have been explored and studied for practical $\mathrm{H}_{2}$ storage but the criteria of the U.S. Department of Energy (DOE) for the year of 2017 have yet to be accomplished. ${ }^{6}$ Thus, it is important to design and explore novel solid-state materials with the desired characteristics to serve as economical, efficient and viable solutions for reversible $\mathrm{H}_{2}$ storage.

Carbon-based nanomaterials, owing to their low cost, light weight, large surface area and novel $\mathrm{H}_{2}$ adsorption, have emerged as a subject of further research interest. ${ }^{7-15}$ However, in their pristine forms such nanomaterials show very feeble binding affinities as a result of weak van der Waals interactions between $\mathrm{H}_{2}$ and the host material. ${ }^{16,17}$ For practical purposes the binding energy of $\mathrm{H}_{2}$ with the respective storage material should be in the

${ }^{a}$ Condensed Matter Theory Group, Department of Physics and Astronomy, Uppsala University, Box 516, SE-75120 Uppsala, Sweden

${ }^{b}$ Applied Materials Physics, Department of Materials and Engineering, Royal Institute of Technology (KTH), SE-100 44 Stockholm, Sweden

${ }^{c}$ Centre for Theoretical and Computational Molecular Science, Australian Institute for Bioengineering and Nanotechnology, The University of Queensland, Brisbane, Qld 4072, Australia.E-mail: t.hussain@uq.edu.au

${ }^{d}$ Centre for Clean Energy and Nano Convergence (CENCON), Hindustan University, Padur, Kelambakkam, Chennai, India

$\dagger$ Electronic supplementary information (ESI) available. See DOI: $10.1039 /$ c6ra24890j range of $-0.2 \mathrm{eV}$ to $-0.6 \mathrm{eV}{ }^{18}$ To achieve this criterion, metal decorated carbon-based nanomaterials have been investigated as potential candidates for $\mathrm{H}_{2}$ storage. The binding affinity could be further tuned by introducing isolated charged sites. However, the bonding mechanism and the binding enthalpy for the interaction of $\mathrm{H}_{2}$ with metal adatom depend on the type of metallic site, the extent of polarizability and on charge localization. It has been shown theoretically that charge transfer between the alkali metal (AM) centers and the host material leaves the AM adatom in the cationic state. The $\mathrm{H}_{2}$ adsorption enthalpy is enhanced due to transfer of charge between $\mathrm{H}_{2}$ and the metallic species. ${ }^{19}$ A rapid decrease in adsorption energy due to increasing temperature has also been reported, which limits the room temperature $\mathrm{H}_{2}$ storage of AM functionalized carbonaceous materials. ${ }^{20-23}$ To enhance the binding aptitude of $\mathrm{H}_{2}$, both alkaline earth metal (AEM) and transition metal (TM) coated materials have been extensively studied in the recent past. ${ }^{26-34}$ Smaller adsorption energies of $\mathrm{H}_{2}$ for AEM than TM decorated storage media have been reported. ${ }^{26}$ Functionalization with TMs leads to strong hybridization between the hydrogen $\sigma$ - or $\sigma^{*}$-orbitals and low-lying empty dorbitals in the TM atoms. ${ }^{19}$ This kind of interaction is typically observed in the Kubas complexes, ${ }^{\mathbf{2 4 , 2 5}}$ and is responsible for enhanced binding affinity towards $\mathrm{H}_{2}$ molecules. However, heavier TMs lead to lower gravimetric density for $\mathrm{H}_{2}$ storage. In addition, clustering is also a crucial problem for TM-doped carbon-based materials, leading to lower storage efficiency. ${ }^{27,29}$ Thus, $\mathrm{H}_{2}$ storage efficiency could be drastically improved by using light TM atoms and further preventing the metal clustering effect.

In this context, Nachimuthu et al. have reported $\mathrm{a}_{2}$ storage capacity of $6.4 \mathrm{wt} \%$ in $\mathrm{Ni}-\mathrm{Ti}-\mathrm{Mg}$ functionalized boron-doped graphene. Their results indicate that the boron content in graphitic materials could successfully hinder the formation of metallic clusters. ${ }^{26,33}$ In contrast, Beheshti et al. suggested that 
substitutional doping of boron atoms in graphene also efficiently prevents $\mathrm{Ca}$ atoms from forming clusters, which is unavoidable in the case of pure graphitic materials. ${ }^{30}$

Recent investigations on boron carbide $\left(\mathrm{BC}_{3}\right)$ nanomaterials in pure, ${ }^{35}$ titanium-doped, ${ }^{32}$ calcium-doped ${ }^{42}$ and lithium-doped ${ }^{34}$ systems have urged the scientific community to unravel the potential of this novel material for the purpose of practical $\mathrm{H}_{2}$ storage. In the present work, we are interested in exploring the $\mathrm{H}_{2}$ storage potential of scandium (Sc) functionalized boron carbide $\left(\mathrm{BC}_{3}\right)$ nanosheets using spin-polarized density functional theory (DFT) calculations. Our investigations include structure analysis, bonding characteristics, charge transfer mechanisms and hydrogenation of functionalized $\mathrm{BC}_{3}$ sheets. The novelty of the present study lies in the fact that even in its pristine form $\mathrm{BC}_{3}$ sheet holds multiple Sc dopants with much higher binding energies than graphene, graphane, h-BN sheet, silicene and many other materials, which make this material superior to these $2 \mathrm{D}$ materials.

\section{Methodology}

The computational analysis of structure, energetics, and charge transfer mechanisms was carried out within the framework of spin-polarized DFT using the Vienna Ab-initio Simulation Package (VASP). ${ }^{36,37}$ For electrons, exchange and correlation interactions were treated with generalized gradient approximation (GGA) as suggested by Perdew-Bruke-Ernzerhof,, ${ }^{38}$ whereas the description of ion-electron interactions was provided by the projector augmented wave method (PAW), which treats the following electronic states as valence: Sc 3p4s3d, B s2p1 and C s2p $2 .{ }^{39}$ For a geometrical description, GGA serves quite well except that the actual energies are underestimated. In order to give an accurate description of the energetics of our systems, we need to account for van der Waals interactions. Therefore, we included the semi-empirical corrections of Grimme ${ }^{40}$ as available in VASP. Owing to the sensitiveness of $\mathrm{H}_{2}$ binding energies with functionalized $\mathrm{BC}_{3} @ \mathrm{Sc}$ systems, we have also compared the performance of DFT-D2 with that of non-local van der Waals functional. For all the computations, the energy cut-off was set to $500 \mathrm{eV}$. Our initial designed system was a $2 \times 2 \times 1$ supercell, which consisted of $8 \mathrm{~B}$ and $24 \mathrm{C}$ atoms. A vacuum thickness of $20 \AA$ was inserted normal to the surface in order to decouple the periodically recurring images. The structures were allowed to relax until the total energy and force converged to $10^{-5} \mathrm{eV}$ per atom and $0.05 \mathrm{eV} \AA^{-1}$ respectively. The Brillouin zone was sampled using a $I$-point scheme with $5 \times 5 \times 1$ mesh for structural relaxation. The density of states calculations with a denser $11 \times$ $11 \times 1$ mesh were performed using the tetrahedron method. The charge transfer mechanism between the metal adatoms and $\mathrm{BC}_{3}$ sheet was investigated using Bader charge analysis. ${ }^{41}$

\section{Results and discussion}

To functionalize $\mathrm{BC}_{3}$ we have carefully selected the lightest transition metal Sc, which could result in a high gravimetric density and adequate binding enthalpy for $\mathrm{H}_{2}$. Boron content in $\mathrm{BC}_{3}$ sheets is expected to hinder clustering of Sc atoms over the sheets. The optimized structure of the $(2 \times 2 \times 1)$ supercell of $\mathrm{BC}_{3}$ is presented in Fig. 1 . The supercell of pristine $\mathrm{BC}_{3}$ monolayer consists of $8 \mathrm{~B}$ and $24 \mathrm{C}$ atoms. The primitive cell of $\mathrm{BC}_{3}$ sheet (marked with dotted lines) consists of $2 \mathrm{~B}$ and $6 \mathrm{C}$ atoms.

\section{Structural analysis}

Structural analysis of optimized $\mathrm{BC}_{3}$ reveals the C-C and $\mathrm{B}-\mathrm{C}$ bond lengths to be $1.42 \AA$ and $1.56 \AA$ respectively, which agree well with previously reported results. ${ }^{42,43}$ (a)

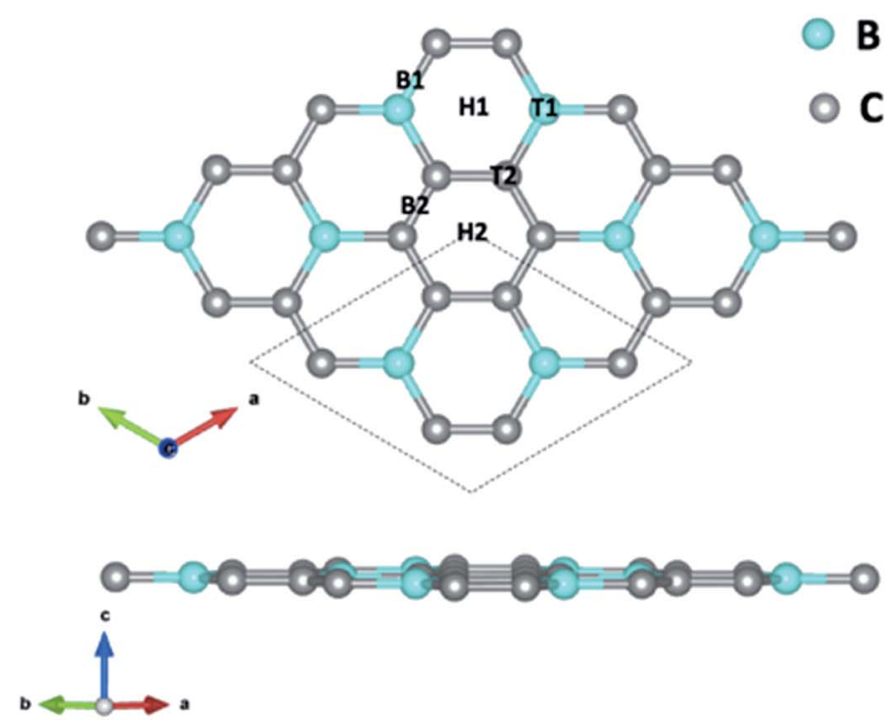

(b)

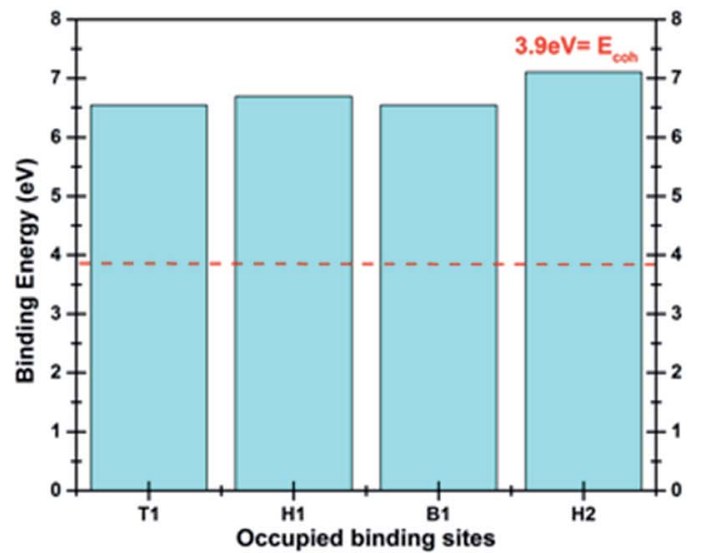

Fig. 1 (a) Top and side view of $\mathrm{BC}_{3}$ sheet; the primitive cell is marked with dotted lines. High symmetry sites available for adatom occupation are indicated as T1, T2, H1, H2, B1 and B2. (b) Binding energy for occupied binding sites. 
To functionalize pure $\mathrm{BC}_{3}$ sheet with $\mathrm{Sc}$ atoms, we have investigated the adsorption behavior of a single $\mathrm{Sc}$ atom on all available high symmetry sites on the single side of the sheet. The six available adsorption sites for the Sc atom are designated (i) top of B atom (T1), (ii) top of C atom (T2), (iii) hollow site in $\mathrm{BC}$ ring (H1), (iv) hollow site in $\mathrm{C}$ ring (H2), (v) bridge between $\mathrm{B}$ and $\mathrm{C}$ atoms (B1) and (vi) bridge between two $\mathrm{C}$ atoms (B2), as shown in Fig. 1. For each possible site, the Sc atom is initially kept at a vertical distance of $2 \AA$. However, the Sc atom initially on $\mathrm{T} 2$ and $\mathrm{B} 2$ sites migrates towards the more favorable $\mathrm{H} 2$ site. The Sc atom binds to $\mathrm{H} 2$ sites with a binding energy of $7.11 \mathrm{eV}$ at a vertical distance $\left(d_{\mathrm{Sc} \text {-sheet }}\right)$ of $1.45 \AA$. Initial and final geometries of all the possible configurations of $\mathrm{Sc}$ on $\mathrm{BC}_{3}$ are given in S1 (ESI $\dagger$ ). The adsorption energies for all Sc occupied sites are presented in Fig. 1(b). The adsorption energy of the Sc atom is defined as:

$$
E_{\mathrm{b}}=\left[E\left(\mathrm{BC}_{3}\right)+E(\mathrm{Sc})\right]-E\left(\mathrm{BC}_{3} @ \mathrm{Sc}\right)
$$

here $E\left(\mathrm{BC}_{3} @ S c\right), E\left(\mathrm{BC}_{3}\right)$ and $E(\mathrm{Sc})$ refer to the total energies of Sc functionalized $\mathrm{BC}_{3}$ sheet, pristine $\mathrm{BC}_{3}$ sheet and $\mathrm{Sc}$ atom respectively. Binding energies of Sc atoms (equivalent to $3.12 \%$ Sc-coverage) on all the available occupation sites show that Sc binds to the sheet with binding energy exceeding the cohesive energy $(3.9 \mathrm{eV})$ of Sc in bulk phase. ${ }^{44}$

The strong binding of single $\mathrm{Sc}$ on $\mathrm{BC}_{3}$ encouraged us to introduce more Sc dopant on the sheet. Thus, in a similar fashion, we found the most favorable site for the second Sc atom (6.25\% Sc-coverage) on the $\mathrm{BC}_{3}$ sheet, as shown in Fig. 2 . The most preferred binding site for the second Sc atom turns out to be the hollow site of the $\mathrm{C}$ ring $(\mathrm{H} 2)$ exactly opposite to the first Sc atom. The binding energy per Sc is found to be $6.02 \mathrm{eV}$, which is slightly lower than for $3.12 \%$ Sc-coverage. However, it is still much higher than Sc-Sc cohesive energy, indicating that $\mathrm{BC}_{3}$ could be further exposed to Sc adatoms. The distance $d_{\mathrm{Sc}-}$ sheet has now increased by $0.19 \AA$ due to the weakening of the bond as compared to the single Sc case (see Table 1).

In the case of $4 \mathrm{Sc}$ on $\mathrm{BC}_{3}$ sheet $(12.5 \%$ Sc-coverage), the most favorable binding configuration is shown in Fig. 2(c). The optimized geometry of this configuration is found to be a little degraded owing to the relatively high Sc doping. Here the binding energy per Sc is found to be $5.45 \mathrm{eV}$, still almost $40 \%$ higher than Sc-Sc cohesive energy. The energetics analysis reveals that even at a significantly high Sc doping concentration, the metal dopants bind strongly enough to nullify cluster formation and make a uniform distribution over the $\mathrm{BC}_{3}$ monolayer.

The bonding mechanism between $\mathrm{Sc}$ and $\mathrm{BC}_{3}$ monolayer could be explained by studying the charge transfer mechanism. We employed Bader charge analysis for this purpose, which depicts a transfer of $1.251 \mathrm{e}^{-}$of charge from $\mathrm{Sc}$ atom to $\mathrm{BC}_{3}$ sheet due to the lower electronegativity of the former than the latter. The bulk portion of this transferred charge goes to the $\mathrm{C}$ atoms of $\mathrm{BC}_{3}$ that are in close vicinity to Sc dopant. This would

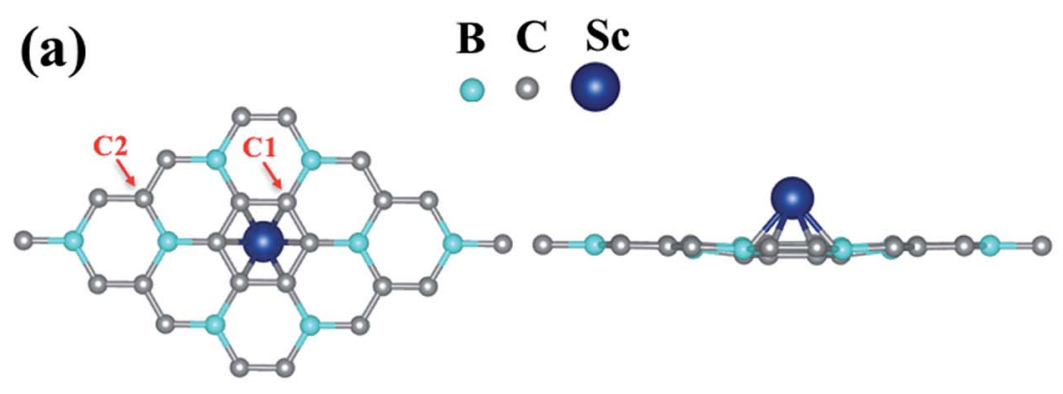

(b)

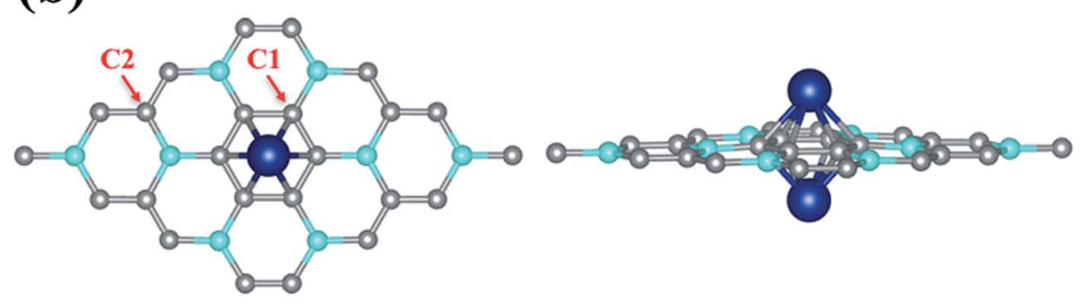

(c)
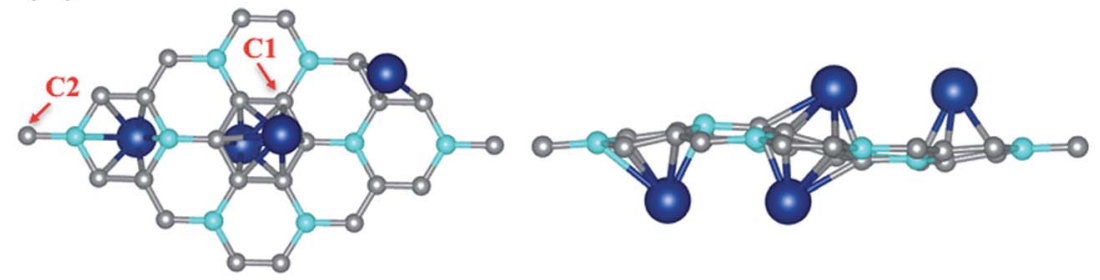

Fig. 2 Top (left) and side (right) views of $\mathrm{BC}_{3}$ sheet functionalized with (a) $1 \mathrm{Sc}$ [3.12\% Sc-coverage], (b) 2 Sc [6.25\% Sc-coverage] and (c) 4 Sc [12.5\% Sc-coverage]. C1 and C2 indicate the carbon atoms that were chosen for the plotting of density of states. 
Table 1 Binding energy $(\mathrm{eV})$ for the most stable configuration, dopant to sheet distance $d_{\mathrm{Sc}-\text { sheet }}$, dopant-dopant distance $d_{\mathrm{Sc}-\mathrm{Sc}}$ and final charge on the dopant

\begin{tabular}{lllll}
\hline System & $\begin{array}{l}\text { Binding energy } \\
\text { per Sc } E_{\mathrm{b}}(\mathrm{eV})\end{array}$ & $\begin{array}{l}\text { Average Sc to } \mathrm{BC}_{3} \text { distance } \\
d_{\mathrm{Sc}-\text { sheet }}(\AA)\end{array}$ & $\begin{array}{l}\text { Minimum dopant-dopant } \\
\text { distance } d_{\mathrm{Sc}-\mathrm{Sc}}(\AA)\end{array}$ \\
\hline $\mathrm{BC}_{3}-1 \mathrm{Sc}$ & 7.11 & 1.45 & - & $\begin{array}{l}\mathrm{Charge} \text { per } \\
\mathrm{Sc}\left(\mathrm{e}^{-}\right)\end{array}$ \\
$\mathrm{BC}_{3}-2 \mathrm{Sc}$ & 6.02 & 1.64 & 3.28 & +1.444 \\
$\mathrm{BC}_{3}-4 \mathrm{Sc}$ & 5.45 & 1.95 & 3.65 & +1.390 \\
\end{tabular}

transform Sc into a partially positive charged state, which proves to be extremely helpful in anchoring the incident $\mathrm{H}_{2}$ molecules. The accumulation and depletion of charges were described by plotting the isosurface charge densities for $3.12 \%$, $6.25 \%$ and $12.5 \%$ Sc-coverage, as shown in Fig. $3(\mathrm{a}-\mathrm{c})$. The charge density can be calculated by the following relation:

$$
\Delta \rho=\rho\left(\mathrm{BC}_{3} @ n \mathrm{Sc}\right)-\rho\left(\mathrm{BC}_{3}\right)-\rho(n \mathrm{Sc})
$$

$$
\{n=1,2,4\}
$$

The minimum distance between Sc atoms $\left(d_{\mathrm{Sc}-\mathrm{Sc}}\right)$ in the case of 2 Sc $(6.25 \%$ coverage) and 4 Sc (12.5\% coverage) doping is found to be $3.28 \AA$ and $3.65 \AA$ respectively. The binding energy per Sc atom decreases, whereas the optimum distance between the $\mathrm{Sc}$ and $\mathrm{BC}_{3}$ sheet increases with the increase in Sc dopant. Though the bond elongation for increased Sc-coverage $\left(d_{\text {Sc-sheet }}\right)$ justifies comparatively weaker binding between the sheet and Sc atoms, the binding is still strong enough to prevent the formation of Sc-Sc clusters. Results from structural and charge analysis are presented in Table 1.

\section{(a)}

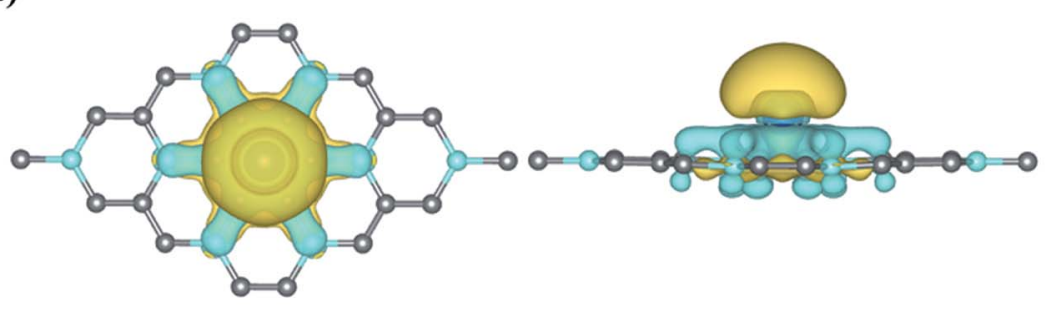

(b)

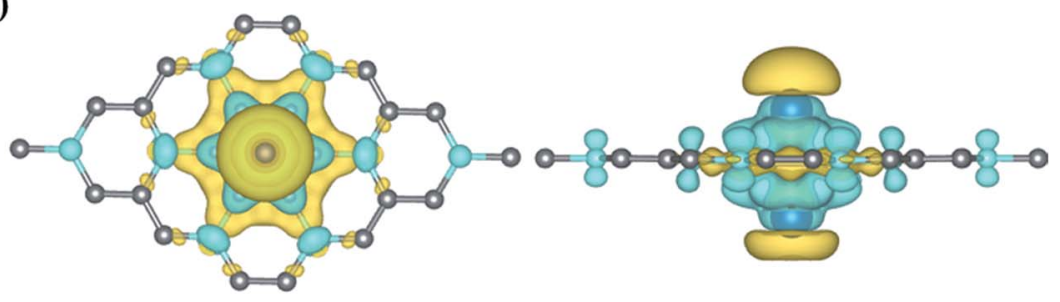

(c)
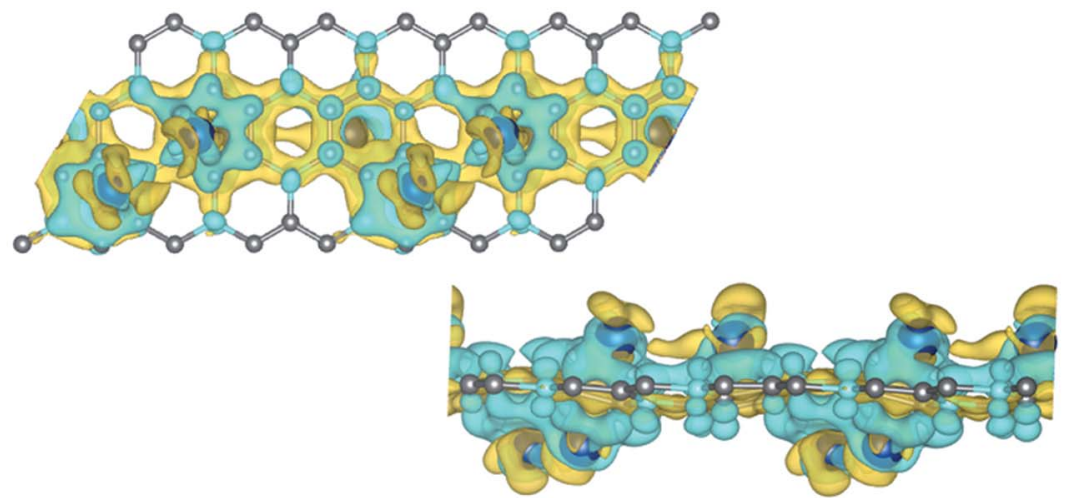

Fig. 3 Top (left) and side (right) views of isosurface charge densities of $\mathrm{BC}_{3}$ with (a) $1 \mathrm{Sc}$ atom [3.12\% Sc-coverage], (b) $2 \mathrm{Sc}$ [6.25\% Sc-coverage] and (c) 4 Sc [12.5\% Sc-coverage]. Cyan and yellow indicate the accumulation and depletion of charge, respectively. 


\section{Electronic structure analysis}

In order to investigate the electronic structure of the designed Sc decorated $\mathrm{BC}_{3}$ system, we have plotted and explained the partial electronic density of states (PDOS) of pure and functionalized $\mathrm{BC}_{3}$ sheets (Fig. 4). PDOS of pure $\mathrm{BC}_{3}$ (Fig. 4(a)) reveals a small bandgap of $\sim 0.65 \mathrm{eV}$, which is in reasonable agreement with the previously reported value. ${ }^{43}$ The PDOS of Sc functionalized $\mathrm{BC}_{3}$ sheet (3.12\% coverage) is shown in Fig. $4(\mathrm{~b})$. $\mathrm{C} 1$ indicates the $\mathrm{C}$ atom that is closer to the Sc atom, and $\mathrm{C} 2$ resides at a greater distance from $\mathrm{Sc}$, as indicated in Fig. 2. The $\mathrm{Sc}(\mathrm{d})$ state is found to be strongly hybridized with $\mathrm{B}(\mathrm{p})$ and $\mathrm{C} 1(\mathrm{p})$ states of the $\mathrm{BC}_{3}$ sheet. With $6.25 \%$ coverage, the extent of $\mathrm{Sc}(\mathrm{d})$ to $\mathrm{B}(\mathrm{p})$ and $\mathrm{C} 1(\mathrm{p})$ hybridization decreases, which is reflected in a decrease in binding energy, as presented in Table 1. With higher coverage, the Sc to Sc attraction weakens the binding energy of $\mathrm{BC}_{3}$ sheet to $\mathrm{Sc}$.

\section{Hydrogenation}

After establishing the stability, the Sc functionalized $\mathrm{BC}_{3}$ sheets were exposed to $\mathrm{H}_{2}$ molecules. We consider placing $\mathrm{H}_{2}$ molecules in different possible configurations whilst performing a set of structural relaxations. The average adsorption energies of $\mathrm{H}_{2}$ were calculated using the following formula:

$$
E_{\mathrm{ads}}=\left[E\left(\mathrm{BC}_{3} @ m \mathrm{Sc}+n \mathrm{H}_{2}\right)-E\left(\mathrm{BC}_{3} @ m \mathrm{Sc}\right)-n E\left(\mathrm{H}_{2}\right)\right] / n
$$

here $E\left(\mathrm{BC}_{3} @ m \mathrm{Sc}+n \mathrm{H}_{2}\right)$ corresponds to the total energy of the hydrogenated system, $E\left(\mathrm{BC}_{3} @ m \mathrm{Sc}\right)$ is the energy of the bare system, $E\left(\mathrm{H}_{2}\right)$ is the energy of $\mathrm{H}_{2}$, and $n$ represents the number of adsorbed $\mathrm{H}_{2}$.

The $\mathrm{H}_{2}$ gravimetric density (storage capacity) can be calculated by the following relation:

$$
\begin{aligned}
\mathrm{H}_{2} \text { storage capacity }= & N_{\mathrm{H}} \times W_{\mathrm{H}} /\left[\left(N_{\mathrm{B}} \times W_{\mathrm{B}}\right)+\left(N_{\mathrm{C}} \times W_{\mathrm{C}}\right)\right. \\
& \left.+\left(N_{\mathrm{Sc}} \times W_{\mathrm{Sc}}\right)+\left(N_{\mathrm{H}} \times W_{\mathrm{H}}\right)\right]
\end{aligned}
$$

In the above relation $N$ represents the number of each type of atoms and $W$ represents the molecular weight of each element present in the system.

The number of $\mathrm{H}_{2}$ molecules around Sc was gradually increased from 1 to the maximum adsorption limit. For the lowest Sc-coverage (3.12\%), a maximum of five $\mathrm{H}_{2}$ molecules per Sc atom could be adsorbed to the functionalized sheet, which resulted in a relatively small $\mathrm{H}_{2}$ storage capacity of $2.364 \mathrm{wt} \%$. Top and side views of the lowest energy hydrogenated systems are represented in Fig. 5(a) whereas all the other possible configurations are given in S2 (ESI $\dagger$ ). The average distance
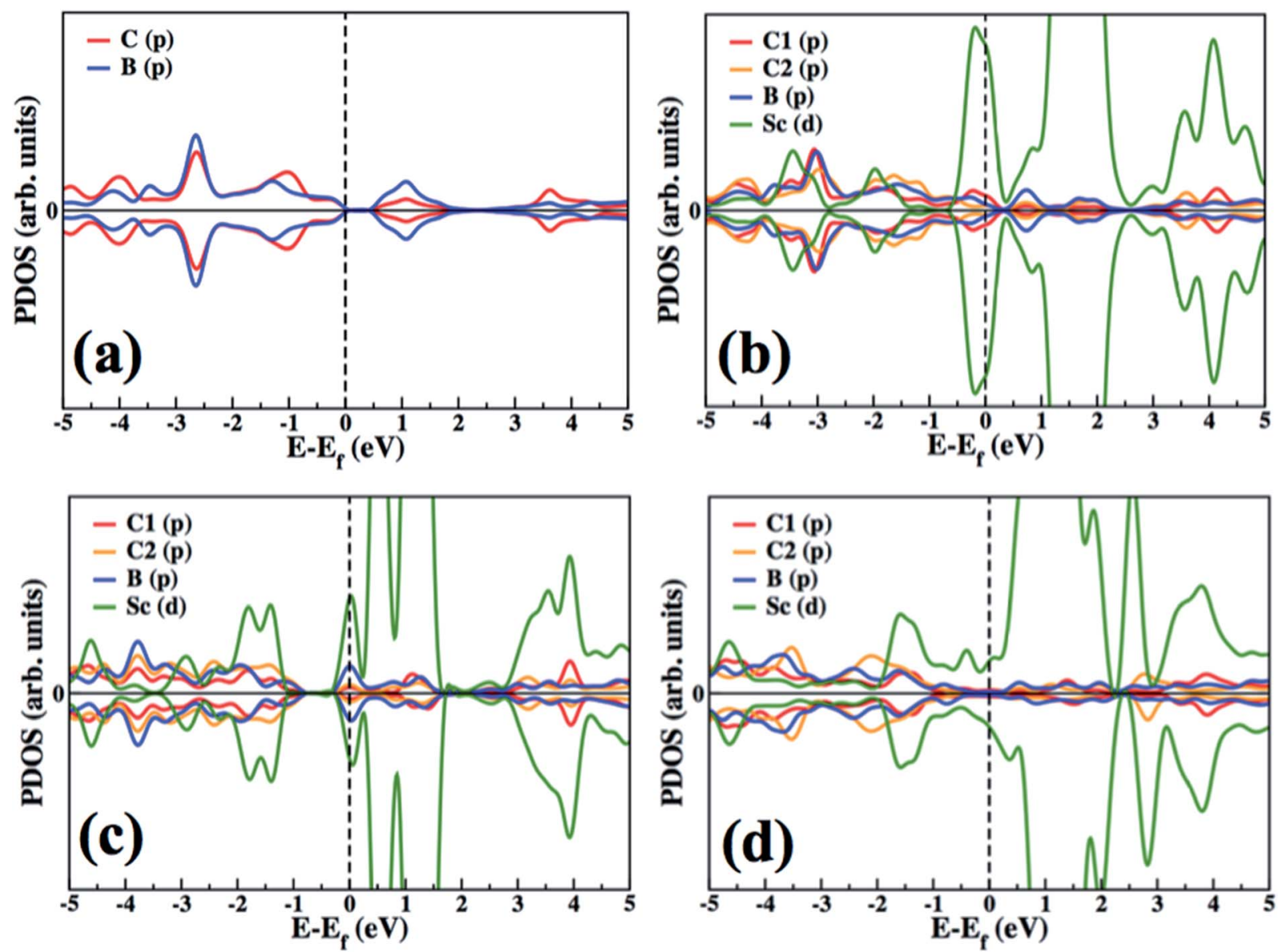

Fig. 4 Partial electronic density of state plots for (a) pure $\mathrm{BC}_{3}$, (b) $\mathrm{BC}_{3}$ with $1 \mathrm{Sc}$ atom $\left[3.12 \% \mathrm{Sc}\right.$-coverage], (c) $\mathrm{BC}_{3}$ with $2 \mathrm{Sc}$ [6.25\% $\mathrm{Sc}$-coverage] and (d) $\mathrm{BC}_{3}$ with $4 \mathrm{Sc}[12.5 \% \mathrm{Sc}$-coverage]. The dashed line indicates the Fermi level. 


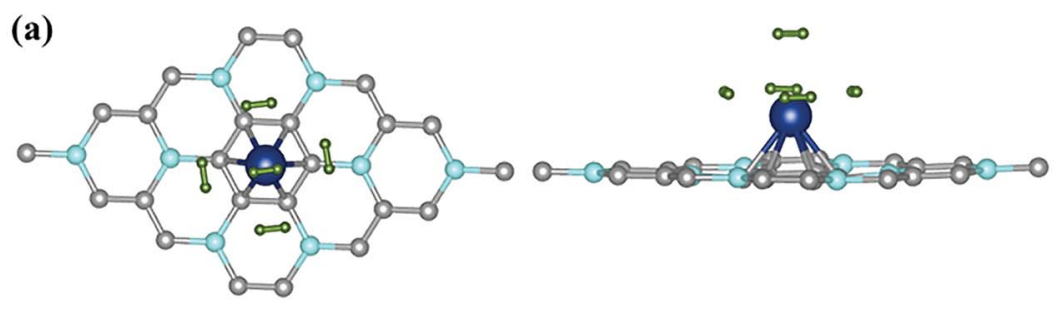

(b)

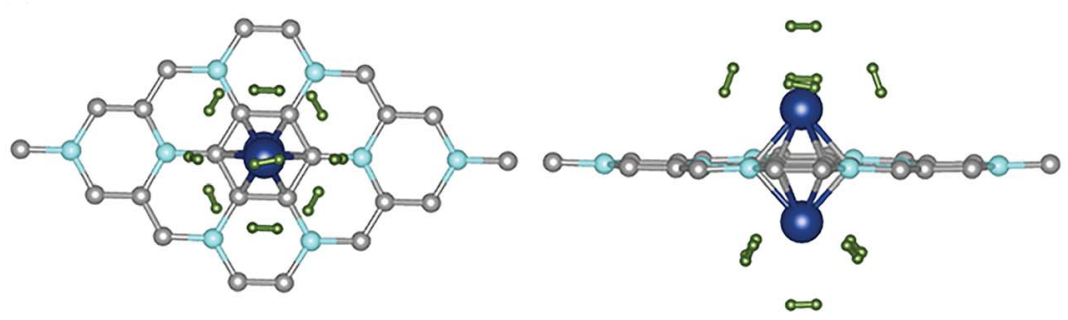

(c)
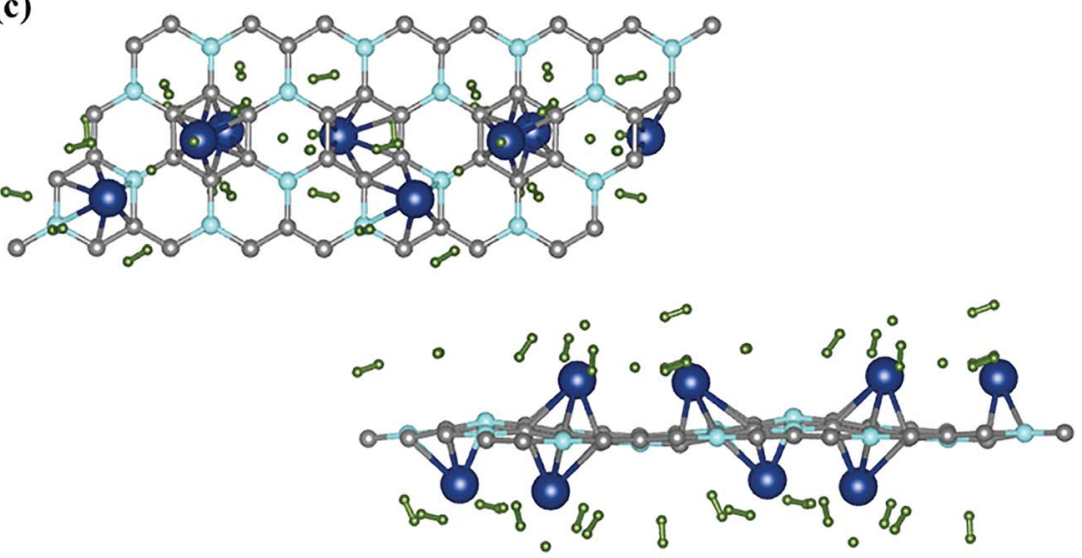

Fig. 5 Hydrogenated $\mathrm{BC}_{3}$ sheets. Top view and side view for (a) $1 \mathrm{Sc}[3.2 \% \mathrm{Sc}$ ] and (b) $2 \mathrm{Sc}[6.4 \%$ Sc]. (c) Extended top and side view for 4 Sc [12.5\% Sc].

between adsorbed $\mathrm{H}_{2}$ and Sc atoms $\left(d_{\mathrm{H}_{2}-\mathrm{Sc}}\right)$ and the average binding energy for maximum $\mathrm{H}_{2}$ uptake are $2.2 \AA$ and $-0.34 \mathrm{eV}$ respectively.

It is important to mention here that the $\mathrm{H}_{2}$ adsorption energies are quite sensitive to van der Waals functionals, so a careful choice of a functional needs to be made in order to achieve unvarying values of adsorption energies. The DFT-D2 method of Grimme used in the present study has been extensively used and it has yielded reliable results consistent with the literature $^{45-48}$ For a comparison we have calculated the adsorption energies of $\mathrm{H}_{2}$ and $2 \mathrm{H}_{2}$ adsorbed on $\mathrm{BC}_{3} @ \mathrm{Sc}$ by using the non-local van der Waals functional optPBe-vdW ${ }^{49}$ and found that the adsorption energies of $\mathrm{H}_{2}$ and $2 \mathrm{H}_{2}$ around $\mathrm{BC}_{3} @ \mathrm{Sc}$ are $-0.310 \mathrm{eV}$ and $-0.293 \mathrm{eV}$ respectively. These values are roughly $23 \%$ lower than those calculated by DFT-D2.

The bond length of $\mathrm{H}_{2}$ has increased slightly to $0.76 \AA$. In the case of two Sc atoms, each Sc could bind $5 \mathrm{H}_{2}$ (shown in Fig. 5(b)), which yielded a $\mathrm{H}_{2}$ storage capacity of $4.184 \mathrm{wt} \%$, significantly higher than that of the $\mathrm{BC}_{3} @ S c$ system. The isosurface charge density of $\mathrm{BC}_{3} @ \mathrm{Sc}-\mathrm{H}_{2}$ and $\mathrm{BC}_{3} @ \mathrm{Sc}-5 \mathrm{H}_{2}$ is shown in $\mathrm{S} 3$ (ESI $\dagger$ ). The adsorption energies of $\mathrm{H}_{2}$ with the functionalized $\mathrm{BC}_{3}$ material again show a decreasing trend with an increasing number of $\mathrm{H}_{2}$. However, the binding energy of $\mathrm{H}_{2}$ for maximum hydrogen coverage is $-0.3 \mathrm{eV}$, which still lies in the energy window above weak physisorption (Fig. 6). The average distance between each $\mathrm{H}_{2}$ and Sc atom is $2.39 \AA$ and the average bond length of $\mathrm{H}_{2}$ is $0.77 \AA$. We employed a similar procedure to hydrogenate the $\mathrm{BC}_{3}$ sheet with $12.5 \%$ Sc-coverage. In total, $16 \mathrm{H}_{2}$ molecules could be incorporated in the functionalized system, giving a gravimetric density of $5.5 \mathrm{wt} \%$ (Fig. 5(c)).

Interestingly, the first $\mathrm{H}_{2}$, introduced in between $2 \mathrm{Sc}$ atoms (on each side of the sheet), experiences dissociative adsorption. The two $\mathrm{H}$ atoms locate to an optimal distance apart $\left(d_{\mathrm{H}-\mathrm{H}}\right)$ of $2.2 \AA$ and each $\mathrm{H}$ attains an electronic charge of $-0.495 \mathrm{e}^{-}$. The bond dissociation leads to adsorption of one $\mathrm{H}$ per Sc atom $(\mathrm{H} /$ Sc) at an average distance ( $\left.d_{\mathrm{H}-\mathrm{Sc}}\right)$ of $1.93 \AA$ and average binding energy of $-0.9 \mathrm{eV}$. Further addition of $\mathrm{H}_{2}$ in the vicinity of Sc atoms leads to associative adsorption of $\mathrm{H}_{2}$. In the first step, the $\mathrm{H}_{2}$ introduced in between $2 \mathrm{Sc}$ atoms gets dissociated due to the presence of enough charge to contribute to the $\sigma^{*}$-antibonding orbitals. To support this argument, Bader charge analysis also reveals partially polarized $\mathrm{H}_{2}$ molecules in the vicinity of Sc atoms. However, for successive adsorption of $\mathrm{H}_{2}$, there is not enough charge available to destabilize the $\mathrm{H}_{2}$ molecule. It is evident from Fig. 6 that for each Sc-coverage value, the average 


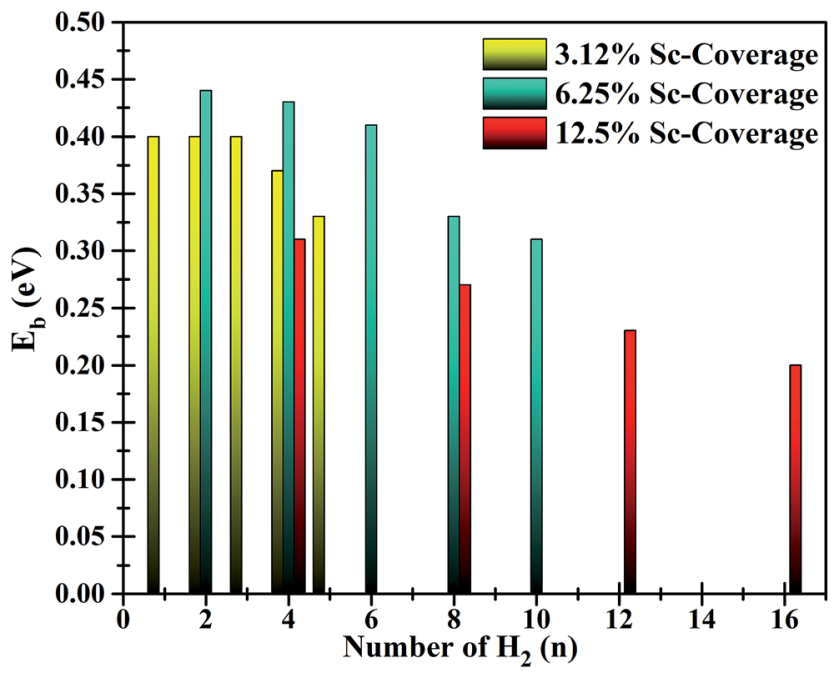

Fig. 6 Adsorption energies of $\mathrm{H}_{2}$ on functionalized $\mathrm{BC}_{3}$ sheets for three different values of Sc-coverage.

adsorption energy of $\mathrm{H}_{2}$ monotonically decreases with increasing number of $\mathrm{H}_{2}$ molecules.

By gradually increasing the number of $\mathrm{H}_{2}$ from $1 \mathrm{H}_{2} / \mathrm{Sc}$ to the maximum limit of adsorption, the average binding energy for associative adsorption varies from -0.31 to $-0.20 \mathrm{eV}$ for $12.5 \%$ Sc-coverage (Fig. 6). The average bond length of adsorbed $\mathrm{H}_{2}$ molecules increased to $0.78 \AA$ which is slightly larger than for free $\mathrm{H}_{2}(0.75 \AA)$. Bond elongation is typically observed in Kubas interaction where an amount of charge is donated from $\mathrm{H}_{2}$ to the empty d-orbital of TM followed by back-donation of charge from TM to an anti-bonding orbital of $\mathrm{H}_{2}$. Such donation and back-donation of charge governed by Kubas interaction has been extensively studied in the literature., ${ }^{8,50-52}$ The greater charge transfer results in a longer bond length of $\mathrm{H}_{2}$. Successive addition of $\mathrm{H}_{2}$ molecules to the functionalized $\mathrm{BC}_{3}$ sheet (with $12.5 \%$ Sc-coverage) reveals that a total of $15 \mathrm{H}_{2}$ (including 2 dissociated molecules) could be adsorbed in the vicinity of Sc atoms, where one $\mathrm{H}_{2}$ directly binds to the sheet at a distance ( $d_{\mathrm{H}_{2} \text {-sheet }}$ ) of $2.7 \AA$ above the $\mathrm{B} 2$ site. Top and side views of the functionalized system with maximum $\mathrm{H}_{2}$ uptake and average adsorption energies with increasing number of $\mathrm{H}_{2}$ are represented in Fig. 5(c).

The functionalized systems ( $\left.\mathrm{BC}_{3} @ \mathrm{Sc}, \mathrm{BC}_{3} @ 2 \mathrm{Sc}, \mathrm{BC}_{3} @ 4 \mathrm{Sc}\right)$ reported in the present work could achieve a maximum $\mathrm{H}_{2}$ storage capacity of $5.5 \mathrm{wt} \%$, which fulfills the DOE target to be met by the year 2017. However, the $\mathrm{H}_{2}$ capacity is smaller than given in a paper by Durgun et al., ${ }^{7}$ which reported on the $\mathrm{H}_{2}$ storage properties of selected transition metals ( $\mathrm{Ti}, \mathrm{Sc}, \mathrm{V}$ ) decorated on carbon chain, graphene and carbon nanotubes, which attained high $\mathrm{H}_{2}$ storage capacities. However, to ensure the uniform distribution of metal dopants over the host materials, the binding energies of the former must be higher than their respective cohesive energies. In the paper mentioned above, the binding energies of TM dopants ( $\mathrm{Ti}, \mathrm{Sc}, \mathrm{V}$ ) fall short of their respective cohesive energies, which could allow the formation of TM clusters which significantly reduces the $\mathrm{H}_{2}$ storage capacity.
The novelty of our work lies in the fact that, despite lower $\mathrm{H}_{2}$ storage capacity as compared with the Durgun et al. paper, Sc, 2 $\mathrm{Sc}$ and $4 \mathrm{Sc}$ bind strongly with $\mathrm{BC}_{3}$, nullifying the possibility of cluster formation and ensuring the formation of stable doped systems.

\section{Conclusions}

We have performed spin-polarized DFT calculations to study the $\mathrm{H}_{2}$ adsorption aptitude of Sc decorated $\mathrm{BC}_{3}$ sheet. Our calculations reveal that $\mathrm{Sc}$ binds strongly to the $\mathrm{BC}_{3}$ sheet with a binding energy that is much higher than its bulk cohesive energy. Hydrogen storage investigations performed with 3 different Sc-coverages $(3.12 \%, 6.25 \%$, and $12.5 \%)$ show that the binding energy of $\mathrm{Sc}$ on pristine $\mathrm{BC}_{3}$ sheet decreases from $7.11 \mathrm{eV}$ to $5.45 \mathrm{eV}$ as the Sc content increases from $3.12 \%$ to the maximum $12.5 \%$. However, even in the case of high Sc doping, the calculated binding energies are still much higher than their corresponding cohesive energies. The density of states analysis shows that $\mathrm{Sc}$ adsorption tunes the electronic behavior of $\mathrm{BC}_{3}$ sheet and metallicity is enhanced with an increased Sc content. Adsorption energies of $\mathrm{H}_{2}$ on $\mathrm{BC}_{3}-\mathrm{Sc}_{n}$ systems lie within an energy window that is reasonable for practical $\mathrm{H}_{2}$ storage. Based on these results we expect that $\mathrm{Sc}$ decorated $\mathrm{BC}_{3}$ sheets can be used as a practical $\mathrm{H}_{2}$ storage medium.

\section{Acknowledgements}

We acknowledge the Swedish Research Council (VR) and StandUp, Carl Trygress Foundation for financial support. SNIC and UPPMAX are acknowledged for providing computing time. We also acknowledge the assistance of resources provided at the NCI National Facility systems at the Australian National University through the National Computational Merit Allocation Scheme supported by the Australian Government and the University of Queensland Research Computing Centre.

\section{References}

1 L. Schlapbach and A. Zuttel, Nature, 2001, 414, 353.

2 G. W. Crabtree, M. S. Dresselhaus and M. V. Buchanan, Phys. Today, 2004, 57, 39.

3 S. W. Jorgensen, Curr. Opin. Solid State Mater. Sci., 2011, 15, 39.

4 K. Mazloomi and C. Gomes, Renewable Sustainable Energy Rev., 2012, 16, 3024.

5 A. W. Van den Berg and C. O. Arean, Chem. Commun., 2008, 6, 668.

6 Targets for Onboard Hydrogen Storage Systems for Light-duty Vehicles, http:/www1.eere.energy.gov/hydrogenandfuelcells/ storage/pdfs/targets_onboard_hydro_storage_explanation.pdf, accessed October 2016.

7 E. Durgun, S. Ciraci and T. Yildirim, Phys. Rev. B: Condens. Matter Mater. Phys., 2008, 77, 085405.

8 T. Yildirim and S. Ciraci, Phys. Rev. Lett., 2005, 94, 175501.

9 W. Liu, Y. H. Zhao, Y. Li, Q. Jiang and E. J. Lavernia, J. Phys. Chem. C, 2009, 113, 2028. 
10 Q. Sun, P. Jena, Q. Wang and M. Marquez, J. Am. Chem. Soc., 2006, 128, 9741.

11 S. A. Shevlin and Z. X. Guo, Chem. Soc. Rev., 2009, 38, 211.

12 M. Yoon, S. Y. Yang, C. Hicke, E. Wang, D. Geohegan and Z. Y. Zhang, Phys. Rev. Lett., 2008, 100, 206806.

13 K. R. S. Chandrakumar and S. K. Ghosh, Nano Lett., 2008, 8, 13.

14 Q. Wang, Q. Sun, P. Jena and Y. Kawazoe, J. Chem. Theory Comput., 2009, 5, 374.

15 K. Takahashi, Y. Wang, S. Chiba, Y. Nakagawa, S. Isobe and S. Ohnuki, Sci. Rep., 2014, 4, 4598.

16 M. Ritschel, M. Uhlemann, O. Gutfleisch, A. Leonhardt, A. Graff, C. Taschner and J. Fink, Appl. Phys. Lett., 2002, 80, 2985.

17 B. Panella, M. Hirscher and S. Roth, Carbon, 2005, 43, 2209. 18 Y.-H. Kim, Y. Zhao, A. Williamson, M. J. Heben and S. B. Zhang, Phys. Rev. Lett., 2006, 96, 016102.

19 R. C. Lochan and M. Head-Gordon, Phys. Chem. Chem. Phys., 2006, 8, 1357.

20 W. Q. Deng, X. Xu and W. A. Goddard, Phys. Rev. Lett., 2004, 92, 166103.

21 G. E. Froudakis, Nano Lett., 2001, 1, 531.

22 Y. L. Zhao, R. Q. Zhang and R. S. Wang, Chem. Phys. Lett., 2004, 398, 62.

23 Z. H. Zhu, G. Q. Lu and S. C. Smith, Carbon, 2004, 42, 2509.

24 G. J. Kubas, Chem. Rev., 2007, 107, 4152.

25 G. J. Kubas, J. Organomet. Chem., 2001, 635, 37.

26 S. Nachimuthu, P.-J. Lai, E. G. Leggesse and J.-C. Jiang, Sci. Rep., 2015, 5, 16797.

27 Q. Sun, Q. Wang, P. Jena and Y. Kawazoe, J. Am. Chem. Soc., 2005, 127, 14582.

28 Y. Suttisawat, P. Rangsunvigit, B. Kitiyanan, M. Williams, P. Ndungu, M. V. Lotototsky and S. Kulprathipanja, Int. J. Hydrogen Energy, 2009, 34, 6669.

29 S. Li and P. Jena, Phys. Rev. Lett., 2006, 97, 209601.

30 E. Beheshti, A. Nojeh and P. Servati, Carbon, 2011, 49, 1561.

31 C. M. Ramos-Castillo, J. U. Reveles, M. E. Cifuentes-Quintal, R. R. Zope and R. de Coss, J. Phys. Chem. C, 2016, 120, 5001.

32 L. Ma, J. Wang, Y. Liang, G. Wang, Chinese Scientific Papers Online, 2015, www.paper.edu.cn/index.php/default/releasepaper/ downPaper/201503-287, accessed October 2016.
33 S. Nachimuthu, P. J. Lai and J. C. Jiang, Carbon, 2014, 73, 132.

34 Z. Yang and J. Ni, Appl. Phys. Lett., 2012, 100, 183109.

35 F.-C. Chuang, Z.-Q. Huang, W.-H. Lin, M. A. Albao and W.-S. Su, Nanotechnology, 2011, 22, 135703.

36 G. Kresse and J. Hafner, Phys. Rev. B: Condens. Matter Mater. Phys., 1993, 47, 558.

37 G. Kresse and J. Hafner, Phys. Rev. B: Condens. Matter Mater. Phys., 1994, 49, 14251.

38 J. P. Perdew, K. Burke and M. Ernzerhof, Phys. Rev. Lett., 1996, 77, 3865.

39 P. E. Blochl, Phys. Rev. B: Condens. Matter Mater. Phys., 1994, 50, 17953.

40 S. Grimme, J. Comput. Chem., 2006, 27, 1787.

41 R. F. W. Bader, Atoms in Molecules: a Quantum Theory, Oxford University Press, Oxford, 1990.

42 Z. Yang and J. Ni, Appl. Phys. Lett., 2010, 97, 253117.

43 D. Tománek, R. M. Wentzcovitch, S. G. Louie and M. L. Cohen, Phys. Rev. B: Condens. Matter Mater. Phys., 1988, 37, 3134.

44 C. Kittel, Introduction to Solid State Physics, Wiley, New York, 7th edn, 1960.

45 T. Hussain, K. Takahashi and D. J. Searles, J. Phys. Chem. A, 2016, 120, 2009.

46 T. Hussain, M. S. Islam, G. S. Rao, P. Panigrahi, D. Gupta and R. Ahuja, Nanotechnology, 2015, 26, 275401.

47 T. Hussain, S. Chakraborty, T. W. Kang, B. Johansson and R. Ahuja, ChemPhysChem, 2014, 16, 634.

48 T. Hussain, A. De Sarkar and R. Ahuja, Int. J. Hydrogen Energy, 2014, 39, 2560.

49 K. Lee, E. D. Murray, L. Kong, B. I. Lundqvist and D. C. Langreth, Phys. Rev. B: Condens. Matter Mater. Phys, 2010, 82, 081101.

50 H. Lee, J. Ihm, M. L. Cohen and S. G. Louie, Phys. Rev. B: Condens. Matter Mater. Phys., 2009, 80, 115412.

51 A. Bhattacharya, S. Bhattacharya, C. Majumdar and G. P. Das, J. Phys. Chem. C, 2010, 114, 10297.

52 C.-G. Zhang, R. Zhang, Z.-X. Wang, Z. Zhou, S. B. Zhang and Z. Chen, Chemistry, 2009, 15, 5910. 